\title{
Zusammenfassung der Forschungsergebnisse und Ausblick
}

\section{Markus Derer}

Ziel dieses Forschungsprojektes war die Entwicklung einer wissenschaftlich fundierten Perspektive hinsichtlich des Einsatzes eines automatisierten Shuttlebusses im Öffentlichen Personennahverkehr (ÖPNV). Die Untersuchungen im Rahmen der Begleitforschung orientierten sich dabei stets an der übergeordneten Forschungsfrage:

„Kann durch die Einführung eines selbstfahrenden/autonomen Shuttles eine Ergänzung des heutigen ÖPNV geschaffen werden oder kann dadurch eine neue Form des ÖPNV geschaffen werden, die bisherige Strukturen ablösen wird?“

Im Rahmen des Pilotbetriebs der DB Regio Bus in Kooperation mit dem Landkreis Rottal-Inn, dem Fahrzeughersteller EasyMile, dem TÜV Süd und der Marktgemeinde in Bad Birnbach gingen die Untersuchungen technologischen, technischen, gesellschaftlichen sowie verkehrsplanerischen Fragestellungen nach.

Die Einwohner und Gäste des Kurorts Bad Birnbach haben seit Oktober 2017 die Möglichkeit, den zu diesem Zeitpunkt neu eingeführten automatisierten Shuttlebus im öffentlichen Regelbetrieb kennenzulernen und zu testen. Daher ist es zunächst von Interesse, ob und wie sich die Mobilitätswahrnehmung der ortsansässigen Bevölkerung dadurch verändert haben könnte. Im Rahmen der von der DB Regio Bus durchgeführten Haushaltsbefragung (Kap. 3, Baniewicz und Neff) konnten hier seit der Einführung des Shuttlebusses keine signifikanten Veränderungen registriert werden. Zum einen ist ein Grund in der überwältigenden Nutzergruppe zu vermuten. Vom zusätzlichen Mobilitätsangebot können Badetouristen - die die überregional bekannte Therme besuchen - am meisten profitieren. Dementsprechend erfährt der Pilotbetrieb die größte Nachfrage durch Touristen und Besucher der Gemeinde Bad Birnbach. Zum anderen vollzieht sich ein Wandel im Mobilitätsverhalten über einen langfristigen Horizont, der die angesetzte Projektlaufzeit vermut-

M. Derer $(\bowtie)$

Technische Hochschule Ingolstadt, Ingolstadt, Deutschland

E-Mail: markus.derer@thi.de 
lich übertrifft. Grundsätzlich hat der Pilotbetrieb aber dazu geführt, dass sich die Bevölkerung mit ihrer eigenen Mobilität und ihrem Mobilitätsverhalten stärker auseinandersetzt und offen für neue Angebote ist.

Eine nachhaltige und attraktivitätssteigernde Integration neuer Mobilitätsangebote, unabhängig von zugrunde liegender Technologie oder Neuartigkeit des Angebots an sich, kann u. a. durch eine ausreichende Nutzung durch Mobilitätsnachfrager realisiert werden. Um das zu erreichen, müssen Innovationen ihren Mobilitätsnachfragern einen spürbaren Mehrwert bieten können. Dieser Fragestellung ging die Deutsche Bahn in Bad Birnbach auf den Grund und analysierte die Veränderungen in der Konnektivität des Bad Birnbacher Verkehrsnetzes (Kap. 4, Jürgens). Die Konnektivität eines Verkehrsnetzes beschreibt dessen Erschließungs- und Verbindungsqualität. Im Zuge der Untersuchung konnte herausgefunden werden, dass der automatisierte Shuttlebus eine Möglichkeit zur Lösung des Erste/Letzte-Meile-Problems, insbesondere in ländlichen Räumen, darstellen kann. Der Pilotbetrieb hat bereits im ersten Jahr einen positiven Einfluss auf die Erreichbarkeit des gesamten Ortes. Des Weiteren ist abzusehen, dass die nächsten Evolutionsstufen des automatisierten Shuttlebusses das Potenzial der Konnektivität des Bad Birnbacher Verkehrsnetzes weiter steigern werden.

Tatsächlich schöpft der automatisierte Shuttlebus sein technisches Potenzial noch nicht aus. Neben dem jetzigen Entwicklungsstand definieren hohe Zulassungsauflagen und entsprechende restriktive Bedingungen systemische Grenzen. Das Forschungszentrum für integrale Fahrzeugsicherheit der Technischen Hochschule Ingolstadt (CARISSMA) kommt im Rahmen seiner Analysen (Kap. 5, Kolb et al.) zu dem Schluss, dass der automatisierte Shuttlebus im aktuellen Zustand für die Teilnahme an einem regulären Serienbetrieb im Öffentlichen Personennahverkehr noch nicht bereit ist. Zu fehlenden Voraussetzungen für eine sichere Teilnahme am örtlichen Mischverkehr kommen herausfordernde Witterungsverhältnisse wie Schneefall, Regen und Nebel hinzu. Allgemein ist die verbaute Sensorik nicht in der Lage, ein ganzheitliches Umfeld mit allen relevanten Informationen konsistent darzustellen, auszuwerten und automatisiert in allen Situationen die richtige Fahrstrategie des Shuttlebusses zu realisieren. Um allen möglichen Herausforderungen, die ein Serienbetrieb mit sich bringt, sicher und zielführend begegnen zu können, benötigt der Shuttlebus folglich eine wesentlich umfangreichere bzw. besser abgestimmte Sensorik. Des Weiteren müssen sicherheitskritische Merkmale weiterhin vorrangig behandelt werden, auch wenn diese in den ersten Entwicklungsstadien Komforteinbußen für die zu befördernden Passagiere zur Folge haben werden. Aus technologischer Sicht hat der anspruchsvolle Zulassungsprozess des TÜVs seinen Zweck erfüllt, da ein bislang unfallfreier Betrieb gewährleistet werden konnte.

Wie wichtig ein restriktiver und ausgereifter Zulassungsprozess hinsichtlich neuer Funktionalitäten im Bereich des autonomen Fahrens ist, zeigen auch die Ergebnisse der Untersuchungen zur gesellschaftlichen Akzeptanz in der Interaktion mit dem Shuttlebus.

Die von der Technischen Hochschule Ingolstadt durchgeführten Studien (Kap. 6 und 7, Wintersberger et al.) zeigen nicht nur einen unmittelbaren Zusammenhang zwischen der artikulierten Akzeptanz und einem Interesse an der Thematik. Knapp zwei Drittel sprachen 
der Technologie bereits zu Beginn ein großes Vertrauen aus, mitunter mit Hinweis auf das verlässliche deutsche Zulassungssystem für motorisierte Fahrzeuge. Des Weiteren hat sich die niedrige Geschwindigkeit, die im Rahmen des Pilotbetriebs sukzessiv erhöht wird, als zielführende Erprobungsstrategie erwiesen. Durch die ausbleibenden Aktionsdynamiken, die eine höhere Betriebsgeschwindigkeit insbesondere in Kurven zur Folge gehabt hätten, konnten sich die Probanden im Erstkontakt an den automatisierten Shuttlebus gewöhnen und vertrauensbildende Erfahrungen machen. Aus diesem Grunde ist ein Fortführen dieser Strategie auch in weiteren Pilotbetrieben zu empfehlen, da sie eine initiale Akzeptanz fördert und eine nachhaltige Vertrauensbasis zwischen dem Menschen und der Maschine darstellt. Gleichermaßen war zu beobachten, dass jüngere Probanden generell ihrem Zeitempfinden eine höhere Relevanz zuschrieben. Für alle Teilnehmer lässt sich sagen, dass hinsichtlich der Betriebsgeschwindigkeit auf die sinnvolle Integration des Shuttlebusses zu achten ist, d. h. die Strecke muss im Vergleich zum bestehenden Mobilitätsangebot vorteilhaft gewählt sein. Eine Teilnahme-Incentivierung erscheint in diesem Kontext sinnvoll, sodass möglichst viele Menschen Erfahrungen durch Benutzen sammeln können. Im potenziellen Serienbetrieb ist dennoch eine höhere Betriebsgeschwindigkeit empfehlenswert, um eine komplementäre und daher nachfragefähige Wirkung zum bestehenden Gesamtsystem ÖPNV realisieren $\mathrm{zu}$ können. Ein langfristig niedrig angesetzter Geschwindigkeitsbereich könnte den durchschnittlichen Verkehrsablauf jedoch beeinträchtigen und der automatisierte Shuttlebus an Attraktivität und Reputation verlieren. Diese Faktoren gilt es auch in der Kommunikation des Shuttlebusses mit dem übrigen Verkehrsgeschehen zu beachten. So fühlte sich beispielsweise ein Drittel der Probanden nicht ausreichend informiert, sodass sporadisch Ungewissheit über die nächsten Aktionen des Shuttlebusses herrschte. Die klassischen Merkmale wie Blinken oder Hupen, die die nächsten Aktionen von Verkehrsteilnehmern nach außen hin kommunizieren, sollten demnach auch bei automatisierten Shuttlebussen - herstellerunabhängig und für alle standardisiert - beibehalten werden.

Diese Erkenntnisse konnten im Rahmen einer Studiendurchführung zu Tage gefördert werden. Insbesondere in einem Kurort wie Bad Birnbach, der jährlich über 110.000 Badeund Kurbesucher empfängt, ist zu beachten, inwieweit die Antworten der befragten Probanden Aufschluss auf die Grundgesamtheit in Deutschland schließen lassen. Beispielsweise haben junge Menschen, die unter anderem als Schüler die ortsansässige Physiotherapie-Schule besuchen, eine andere Wahrnehmung des Shuttlebusses artikuliert als nicht-einheimische Touristen. Um in diesem Kontext die gewonnenen Erkenntnisse bestätigen zu können, sind weitere Untersuchungen mit definierten Zielgruppen in ähnlichen Pilotbetrieben erforderlich.

Wie bereits erwähnt, entscheiden nicht nur Akzeptanz und Nachfrage der Bevölkerung über Erfolg und Misserfolg neuer Technologien. Vielmehr entstehen Wechselwirkungen zwischen zahlreichen Akteur(gruppe)n aus unterschiedlichen gesellschaftlichen Teilbereichen (Politik, Verwaltung, Privatwirtschaft, Zivilgesellschaft) und dem Artefakt der neuen Technologie, die als gesellschaftliche Einbettungsprozesse beschrieben werden können. D. h. nicht nur die Technologie passt sich den Nachfrage- oder Marktmustern an, sondern 
auch die Umwelt, also bspw. Infrastrukturen, rechtliche Rahmenwerke, Zuständigkeiten oder öffentliche Meinungen/Diskurse, passen sich den Innovationen an und können so zu einer gesellschaftlichen Legitimation oder ggf. Blockade von Neuerungen beitragen. Die Julius-Maximilians-Universität Würzburg setzte sich mit diesem Bereich auseinander (Kap. 8, Appel et al.). Auf Basis der durchgeführten Untersuchungen kann gezeigt werden, dass die Befürwortung und proaktive Unterstützung durch beteiligte Akteure aus Politik, Gesetzgebung, Bevölkerung und Privatwirtschaft als Gesamtheit eine zwingende Voraussetzung für die erfolgreiche Umsetzung des Pilotbetriebs darstellt und erst dadurch die Finanzierung des Projekts sowie die Organisation von Zuständigkeiten gewährleistet werden konnten. Hinsichtlich der gesellschaftlichen Einbettungsprozesse wurde gezeigt, dass Phasen der internen Einbettung in unternehmerische und organisatorische Umfelder gefolgt wurden von Phasen der externen Einbettung in ein Regelungsumfeld und eine breitere Öffentlichkeit. Auch wenn bislang nicht von einer disruptiven Einbettung autonomer Kleinbusse in gesellschaftliche Kontexte gesprochen werden kann, konnten zahlreiche Erfahrungen über die Wechselwirkungen der Technologie mit den unterschiedlichen gesellschaftlichen Teilbereichen gesammelt werden. Zukunftsprognosen hinsichtlich des langfristigen Einsatzzwecks des automatisierten Shuttlebusses können demnach aber nicht verbindlich formuliert werden, da gesellschaftliche Diskurse und Erfahrungen noch nicht gefestigt sind.

Auf Grundlage einer weiterführenden Haushaltsbefragung (Kap. 9, Rauh et al.) werden Gründe dafür im Innovationsgrad, in fehlender Erfahrung und fehlendem Kontakt mit dem automatisierten Shuttlebus als nutzbarem Verkehrsmittel vermutet. Beispielsweise argumentierten Befragte, dass das Testgebiet in Bad Birnbach zu klein sei und daher lediglich eingeschränkte Aussagekraft hinsichtlich möglicher Entwicklungen in der Zukunft besäße. Neben Zuverlässigkeit und Sicherheit konnte auch der Faktor Erlebnischarakter gut abgefragt und eingeschätzt werden. Trotzdem erscheint den Befragten die Wettbewerbsfähigkeit des automatisierten Shuttlebusses im Vergleich zu anderen konventionellen Verkehrsmitteln eingeschränkt. Diese Einschätzung ist mitunter auf die niedrige Einsatzgeschwindigkeit und vereinzelte Probleme während des Betriebs zurückzuführen. Im Rahmen dieses Projekts konnte ausschließlich die gegenwärtige Akzeptanzsituation identifiziert werden, die keinen validen Rückschluss auf die Gesamtheit zulässt. Aus diesem Grund ist eine Fortführung dieser Form von Akzeptanzuntersuchungen begleitend zu vergleichbar gearteten Pilotprojekten empfehlenswert. Die Aussagekraft und Vielfältigkeit individueller Präferenzen steigt mit der Zahl an durchgeführten Untersuchungen zur gesellschaftlichen Akzeptanz von automatisierten Shuttlebussen als öffentlichem Mobilitätsangebot.

Die Technische Hochschule Deggendorf nutzte die im Zuge des Pilotbetriebs gewonnenen Parameter, um die Übertragbarkeit des Shuttlebus-Betriebs in andere Regionen zu untersuchen (Kap. 10, Wuth und Dorner). Dabei wurden statische Faktoren wie Straßensteigung und Deckenhöhe von Tunnelunterführungen, aber auch dynamische Parameter wie Fußgänger und andere Verkehrsteilnehmer berücksichtigt. Ziel war dabei eine möglichst vollständige Erfassung des Straßenraums. Auf Grundlage der Datenanalyse dieser Faktoren wurden 
124 Strecken identifiziert, die allein im Bundesland Bayern potenzielle Routen für den Einsatz des automatisierten Shuttlebusses darstellen können. Eine vollständige und detaillierte Abbildung des Straßenraums ist momentan und größtenteils jedoch nicht frei zugänglich. Verfügbare Geobasisdaten besitzen in der Regel nicht die erforderliche und durchgängige Datenqualität, die für ein verwertbares virtuelles Datenmodell unabdingbar ist.

Die Übertragbarkeit kann indes der Impuls für einen essenziellen Brückenschlag von unserem Hier und Jetzt in die manchmal unwirklich erscheinenden Zukunftsszenarien sein. Visionen bilden zwar eine wichtige Grundlage, die als Orientierungshilfe dienen kann. Sie sagen in der Regel aber nichts über den Weg aus, der zu ihnen führt. Mit der Übertragbarkeit wurden neue Einsatzfelder für einen Regelbetrieb des automatisierten Shuttlebusses identifiziert. Diese sind mit der Möglichkeit gleichzustellen, sich weitergehend mit dem Einsatz des Shuttlebusses auseinanderzusetzen und weitere Erkenntnisse gewinnen zu können.

Die Erkenntnisse der einzelnen Forschungsgruppen stellen dabei kleine, aber wesentliche und erfolgsentscheidende Schritte auf dem Weg zu einem nachhaltigen und langfristig zukunftsfähigen Mobilitätskonzept dar. Im Fokus der wissenschaftlichen Untersuchungen lag dabei die gesamtheitliche Betrachtung von Kunde, Produkt, Mobilitätsdienstleister und gesellschaftlichen Kontexten.

Die Konvergenz zwischen dem Menschen, der Technik und der Bedienungsform ist dabei zwingende Voraussetzung für einen erfolgreichen ÖPNV. Von ihr hängt letzten Endes auch die Technologiedurchdringung des Autonomen Fahrens ab. Die Forschungsbereiche des hier vorgestellten Sammelbandes zeigen die Vielfalt und Bandbreite an Fragestellungen, die ein Betrieb eines automatisierten Shuttlebusses mit sich bringt. Ob und wie schnell sich das Autonome Fahren als bewährte Form der Fortbewegung etablieren wird, kann aus heutiger Sicht aber nicht zuverlässig bestimmt werden. 
Open Access Dieses Kapitel wird unter der Creative Commons Namensnennung 4.0 International Lizenz (http://creativecommons.org/licenses/by/4.0/deed.de) veröffentlicht, welche die Nutzung, Vervielfältigung, Bearbeitung, Verbreitung und Wiedergabe in jeglichem Medium und Format erlaubt, sofern Sie den/die ursprünglichen Autor(en) und die Quelle ordnungsgemäß nennen, einen Link zur Creative Commons Lizenz beifügen und angeben, ob Änderungen vorgenommen wurden.

Die in diesem Kapitel enthaltenen Bilder und sonstiges Drittmaterial unterliegen ebenfalls der genannten Creative Commons Lizenz, sofern sich aus der Abbildungslegende nichts anderes ergibt. Sofern das betreffende Material nicht unter der genannten Creative Commons Lizenz steht und die betreffende Handlung nicht nach gesetzlichen Vorschriften erlaubt ist, ist für die oben aufgeführten Weiterverwendungen des Materials die Einwilligung des jeweiligen Rechteinhabers einzuholen. 